\title{
Características definidoras da Resposta Disfuncional ao Desmame Ventilatório como indicadores de acurácia do desmame ventilatório
}

\author{
Defining characteristics of the Dysfunctional Ventilatory Weaning Response as \\ indicators of accuracy of ventilatory weaning \\ Características definitorias de la Respuesta Disfuncional al Destete Ventilatorio \\ como indicadores de precisión del destete ventilatorio
}

\begin{abstract}
Marcos Antônio Gomes Brandão', Fernanda Avellar Cerqueira", Ligia Neres Matos"', Juliana Faria Campos', Mauricio Abreu Pinto Peixoto ${ }^{\mathrm{IV}}$, Cândida Caniçali Primov

' Universidade Federal do Rio de Janeiro, Escola de Enfermagem Anna Nery, Departamento de Enfermagem Fundamental. Rio de Janeiro-RJ, Brasil.

"Ministério da Saúde, Hospital Federal da Lagoa. Rio de Janeiro-RJ, Brasil.

"'Ministério da Saúde, Instituto Nacional de Cardiologia. Rio de Janeiro-RJ, Brasil.

"v Universidade Federal do Rio de Janeiro, Núcleo de Tecnologia Educacional para a Saúde. Rio de Janeiro-RJ, Brasil.

${ }^{\vee}$ Universidade Federal do Espírito Santo, Curso de Enfermagem. Vitória-ES, Brasil.
\end{abstract}

\author{
Submissão: 16-04-2014 Aprovação: 17-07-2014
}

\begin{abstract}
RESUMO
Objetivou-se avaliar as características definidoras do diagnóstico Resposta Disfuncional do Desmame Ventilatório, como indicadores de acurácia das tentativas de desmame. Estudo observacional de 38 eventos de tentativa de desmame ventilatório em pacientes adultos internados em terapia intensiva. Para as características definidoras foram calculadas: sensibilidade, especificidade, valores preditivos positivos e negativos, acurácia ou sensibilidade, razão de verossimilhança e razão de chances diagnóstica. Também foram consideradas as medianas do número de características definidoras nos eventos de sucesso e insucesso. Foram consideradas acuradas: agitação, deterioração nos gases sanguíneos arteriais em relação aos parâmetros basais, uso moderado da musculatura acessória da respiração, aumento da frequência respiratória em relação aos parâmetros basais e frequência respiratória aumentada de forma significativa em relação aos parâmetros basais. Houve diferença estatística nas medianas do número de características definidoras observadas. Conclui-se que a característica definidora e o número delas influenciariam o sucesso da decisão sobre o desmame.
\end{abstract}

Descritores: Avaliação em Enfermagem; Diagnóstico de Enfermagem; Desmame do Respirador.

\section{ABSTRACT}

The study aimed to analyze the defining characteristics of the Dysfunctional Ventilatory Weaning Response as an indicator of the accuracy of ventilatory weaning. Observational study of 38 events of ventilatory weaning in adult patients admitted to intensive care. For the defining characteristics, it was calculated: sensitivity, specificity, positive and negative predictive values, accuracy or efficiency, likelihood ratio positive and negative, and diagnostic odds ratio. It was also considered the median number of defining characteristics in the event of success and failure. It was considered accurate: agitation, deterioration in arterial blood gases from baseline parameters, moderate use of accessory muscles of respiration, increased respiratory rate from baseline parameters and respiratory rate increases significantly with respect to baseline parameters. There was statistical difference in the median number of defining characteristics observed. It was concluded that the defining characteristic and the number of them would influence the success of the weaning decision.

Key words: Nursing Assessment; Nursing Diagnosis; Ventilator Weaning. 


\section{RESUMEN}

El estudio tuvo como objetivo evaluar las características que definen el diagnóstico de Respuesta Disfuncional al Destete Ventilatorio como indicador de la exactitud del destete ventilatorio. Estudio observacional de 38 eventos de destete ventilatorio en pacientes adultos ingresados en cuidados intensivos. Para las características definitorias se calcularon: sensibilidad, especificidad, valores predictivos positivos y negativos, precisión o sensibilidad, cocientes de probabilidad y odds ratio diagnóstica. Fueran consideradas las medianas del número de características definitorias en casos de éxito o de fracaso. Se consideraron precisas: agitación, deterioro de los parámetros de gases en sangre arterial desde la línea de base, uso moderado de los músculos accesorios de la respiración, aumento de la frecuencia respiratoria a partir de parámetros de línea de base y frecuencia respiratoria aumentada significativamente en comparación con los parámetros de línea de base. Hubo diferencia estadísticamente significativa en la mediana del número de características definitorias observadas. Se concluye que la característica definitoria y el número de ellas influyen en el éxito de la decisión sobre el destete ventilatorio.

Palabras clave: Evaluación en Enfermería; Diagnóstico de Enfermería; Desconexión del Ventilador.

\section{AUTOR CORRESPONDENTEＭarcos Antonio Gomes BrandãoＥ-mail: marcosbrandao@ufrj.br}

\section{INTRODUÇÃO}

A Resposta Disfuncional ao Desmame Ventilatório (RDDV) é um diagnóstico de enfermagem definido como a "incapacidade de ajustar-se a níveis diminuídos de suporte ventilatório mecânico, que interrompe e prolonga o processo de desmame ${ }^{\prime \prime(1)}$.

Nos Estados Unidos da América em torno de 800.000 pacientes requerem, por ano, a ventilação mecânica. Na literatura brasileira não foram encontradas estatísticas semelhantes sobre os pacientes que necessitam de ventilação; contudo, estudo realizado no país em 2006 revela que a taxa de ventilação correspondia a 55,6\% dos pacientes internados em terapia intensiva(2). A ocorrência, as exigências de monitoramento e as complicações apontam as respostas ao desmame ventilatório como relevantes na prática de saúde ${ }^{(3)}$.

Estima-se que, para elaborar um diagnóstico mais acurado de insucesso na tentativa de desmame, o enfermeiro precisará de evidências que indiquem o número mínimo de achados característicos do quadro, o valor clínico de cada sinal e sintoma e o intervalo de tempo para sua manifestação. Essas seriam as três principais informações para um diagnóstico sustentado nas informações fornecidas pelas características definidoras (sinais e sintomas).

Notadamente, os dados disponíveis para o diagnosticador interferem no julgamento diagnóstico, onde diferentes gradações de importância podem ser designadas pelo enfermeiro ${ }^{(4)}$. A estimativa exclusivamente baseada na experiência profissional possui limites de confiabilidade clínica. Assim, os diagnósticos de enfermagem precisam ser validados pelas pesquisas que tenham foco na acurácia dos indicadores clínicos para ter notado valor ${ }^{(4)}$.

Se por um lado, a taxonomia da NANDA Internacional apresenta uma lista extensa de sinais e sintomas (características definidoras) do diagnóstico RDDV, ela não indica o valor clínico das características definidoras na predição do evento, nem a sua provável distribuição temporal ou o número mínimo de características definidoras indicativo da presença do diagnóstico.

O problema central desse estudo é o de que não há clareza acerca do valor das características definidoras propostas pela NANDA Internacional para o diagnóstico RDDV como indicadores acurados para a estimativa do fracasso da tentativa de desmame.

Entende-se que a acurácia dos indicadores clínicos da RDDV deva ser investigada por meio de três abordagens às suas características definidoras: (1) como variável de teste diagnóstico para estimar o sucesso/insucesso do desmame, (2) o número mínimo de características a considerar, e (3) a distribuição temporal da característica no fenômeno diagnóstico. Acerca da distribuição temporal das características definidoras da RDDV, estudo já realizado tratou dos elementos temporais ${ }^{(5)}$, assim, permanece a justificativa de investigar as duas primeiras abordagens.

Baseados nos aspectos considerados, este estudo tem como objetivo avaliar as características definidoras do diagnóstico Resposta Disfuncional do Desmame Ventilatório como indicadores de acurácia das tentativas de desmame ventilatório.

\section{METODOLOGIA}

Estudo observacional, realizado com uma amostra de 38 eventos de tentativa de desmame ventilatório de 14 adultos internados por causas clínicas no Centro de Terapia Intensiva de um hospital especializado em cardiologia na região Sudeste do Brasil. Os participantes estavam em ventilação mecânica invasiva por mais de 24 horas, ficando a decisão de início do desmame ventilatório a cargo da equipe multiprofissional de saúde do hospital.

O protocolo institucional de desmame iniciava-se pela manhã e deveria ter a primeira avaliação de tolerância realizada após 30 minutos. Se nenhuma evidência de suspensão fosse verificada, as avaliações posteriores eram realizadas a cada hora até tolerância ou intervalo de 06 horas fora da prótese ventilatória. No caso de falha, nova tentativa seria feita à tarde.

O desfecho do insucesso da tentativa foi a decisão profissional de interrompê-la, baseando-se nos critérios institucionais: saturação de oxigênio menor do que 92\%, frequência respiratória maior do que 35 incursões respiratórias por minuto (irpm), volume corrente menor do que $5 \mathrm{ml} / \mathrm{Kg}$ de peso, uso da musculatura acessória, presença de sudorese, aumento da frequência cardíaca ou pressão arterial média ou queda de $20 \%$ ou mais da linha de base. 
A coleta de dados ocorreu pelo período de quatro meses no ano de 2010 e incluiu as 37 características definidoras apontadas pela NANDA Internacional ${ }^{(1)}$, organizadas conforme a gravidade. A coleta foi realizada por uma das pesquisadoras com a colaboração da enfermeira da equipe de assistência ventilatória da instituição ou da enfermeira designada ao paciente. Aplicou-se a observação contínua, não participante e estruturada à beira do leito durante todo o episódio de tentativa de desmame. Tinha início com o desacoplamento da prótese do paciente e findava com o retorno do mesmo à prótese ou a sua retirada definitiva.

Os enfermeiros foram capacitados para avaliação das características definidoras da RDDV. Testes-piloto foram realizados com as entrevistadoras de modo a verificar a capacidade de concordância entre os observadores e verificar a adequação do instrumento de coleta de dados. A coleta de dados foi iniciada a partir do sucesso dos procedimentos preparatórios e após aprovação em 09/12/2009 no Comitê de Ética em Pesquisa da instituição, registro $n^{0} 326$.

Para análise da acurácia das características definidoras a fim de predizer a resposta ao desmame foram usadas medidas utilizadas em estudos semelhantes ${ }^{(6-8)}$ : sensibilidade (SE), especificidade (ES), valores preditivos positivos (VPP) e negativos (VPN), acurácia ou eficiência (EF), razão de verossimiIhança positiva $(R V+)$ e negativa ( $R V-)$ e razão de chances diagnóstica (RCD) e a acurácia ou eficiência para cada uma das características definidoras identificadas. Foram utilizados os softwares estatísticos Instat Graphpad ${ }^{\circledast}$ e o DAG Stat ${ }^{\circledast}$.

Trazidas as principais medidas de acurácia para o campo do diagnóstico de enfermagem, a sensibilidade representa a probabilidade de se identificar, de forma correta, a presença de um indicador clínico em indivíduos com o diagnóstico de enfermagem. Já a especificidade é a probabilidade de corretamente identificar a ausência do indicador clínico em indivíduos sem o diagnóstico de enfermagem. O valor preditivo positivo (VPP ou $\mathrm{VP}+$ ) representa a probabilidade de se ter o diagnóstico de enfermagem naqueles que possuem a característica definidora. $\mathrm{O}$ valor preditivo negativo (VPN ou VP-) é medida representativa da probabilidade da ausência do diagnóstico de enfermagem em indivíduos sem a característica definidora(7).

Para a verificação do número mínimo de características definidoras identificadas nas situações de sucesso e de insucesso foi aplicada a comparação entre as diferenças das medianas dos dois grupos, e para isso utilizou-se o teste não paramétrico de Mann-Whitney.

A significância estatística das relações das medidas calculadas na tabela de contingência $2 \times 2$ foi calculada aplicando-se o teste exato de Fischer com p bicaudal. Utilizou-se o valor mínimo da Razão de Chances (RC) no intervalo de confiança de 95\% (IC 95\%) como critério de significância clínica das características definidoras.

\section{RESULTADOS}

Foram acompanhadas as tentativas de desmame ventilatório em 14 pacientes. A maioria dos participantes da pesquisa estava compreendida no intervalo de idade de 71 a 90 anos
$(78,5 \%)$ com relativo equilíbrio entre os sexos masculino $(53 \%)$ e feminino $(47 \%)$. O percentual de distribuição entre os sexos é compatível com o de estudo epidemiológico desenvolvido em pacientes com ventilação mecânica no Brasil (2).

As causas das internações hospitalares mais frequentes na amostra foram: Doença Pulmonar Obstrutiva Crônica descompensada, Sepse Pulmonar e Acidente Vascular Encefálico Isquêmico. As demais causas foram de ordem cardiovascular, urinária e oncológica.

Dentre as comorbidades verificaram-se as ocorrências de Hipertensão Arterial Sistêmica, Hipotireoidismo e Insuficiência Renal Crônica.

Todos os participantes do estudo estavam na modalidade de Pressão Positiva Contínua nas Vias Aéreas (CPAP) antes da retirada da prótese ventilatória, sem uso de sedativos, o que permitiu a adequada avaliação do nível de consciência, e sob ventilação mecânica prolongada, o que segundo consenso, ocorreria quando se está na condição há mais de três semanas $^{(9)}$.

Quanto ao protocolo de desmame, o insucesso ocorreu em $47,3 \%$ (18 eventos) do total de observações. Os bem-sucedidos foram 52,6\% (20 eventos), dentre os quais em $26,3 \%$ (10 eventos) não foram identificadas nenhuma característica definidora compatível com a RDDV.

Os principais motivos alegados pelos profissionais para decidir pela interrupção da tentativa de desmame foram motivos associados ou simples. Entre as associações: desconforto ao respirar e inquietação (5,5\%); diminuição da oximetria e hipertensão arterial (5,5\%); agitação e fadiga (5,5\%). Dentre os motivos simples mais prevalentes: a diminuição da oximetria $(27,7 \%)$; e o desconforto ao respirar $(11,1 \%)$. Os demais motivos simples perfazendo 5,5\% dos casos cada: agitação; hipertensão arterial; hipotensão arterial; instabilidade hemodinâmica; piora do aspecto da secreção respiratória, taquicardia; taquipneia; uso da musculatura acessória.

Considerando as situações de sucesso e insucesso das tentativas de desmame ventilatório, foram calculadas as medianas do número de características definidoras nos eventos e relacionadas com a classificação de grave, moderada e leve, conforme proposição da NANDA - Internacional, demonstradas na Tabela 01.

As diferenças verificadas entre as medianas das características definidoras identificadas nos fracassos (9,5 características) e sucesso do desmame (3,5 características) foram consideradas estatisticamente significativas. Apontam que o maior número de características definidoras nos casos de insucesso do desmame não ocorreu ao acaso.

Para a gravidade, a características graves e moderadas foram as que indicaram diferenças entre as medianas com valor de significância. O mesmo não ocorreu com as leves.

As características definidoras com frequência superior a $50 \%$ foram: "A frequência respiratória aumenta de forma significativa em relação aos parâmetros basais" (63,1\%); "Secreções audíveis nas vias aéreas" e "Aumento da frequência respiratória em relação aos parâmetros basais ( $<5$ respirações/ min)", ambas com 52,6\%. As demais características definidoras estão na Tabela 2 . 
Tabela 1 - Medianas do número de características definidoras (CDs) nos grupos de eventos em que houve sucesso e insucesso nas tentativas de desmame, Rio de Janeiro-RJ, 2014

\begin{tabular}{cccc}
\hline Categoria das CDs & Mediana no sucesso & Mediana no insucesso & Valor de p \\
\hline Graves & $1.0(0-5)$ & $4.0(4-7)$ & $0.0003^{*}$ \\
Moderadas & $1.5(0-6)$ & $5.0(3-9)$ & $0.0008^{*}$ \\
Leves & $0.5(0-4)$ & $1.5(0-5)$ & 0.3727 \\
Total & $3.5(0-13)$ & $9.5(6-17)$ & $0.0031^{*}$ \\
\hline
\end{tabular}

* Significância estatística no teste de Mann-Whitney, $p<0.05$.

Tabela 2 - Características definidoras do diagnóstico Resposta Disfuncional ao Desmame Ventilatório, Rio de Janeiro-RJ, 2014

\begin{tabular}{lll}
\hline Características Definidoras & n & $\%$ (IC 95\%) \\
\hline
\end{tabular}

A frequência respiratória aumenta de forma significativa em relação aos parâmetros basais

$24 \quad 63.1(47.3-76.6)$

Secreções audíveis nas vias aéreas; Aumento da frequência respiratória em relação aos parâmetros basais ( $<5$ respirações/min)

$20 \quad 52.6(37.3-67.5)$

Aumento moderado da frequência cardíaca em relação aos parâmetros basais ( $<20$ batimentos/min)

Desconforto ao respirar

$34.2(21.2-50.1)$

Aumento da frequência cardíaca em relação aos parâmetros basais ( $>20$ batimentos/min)

Ruídos adventícios na respiração; Entrada de ar diminuída à ausculta; Uso moderado da musculatura acessória da respiração; Inquietação

Respiração superficial; Aumento moderado da frequência respiratória em relação à linha de base

Agitação; Aumento da concentração na respiração

Deterioração nos gases sanguíneos arteriais em relação aos parâmetros basais

Aumento da pressão sanguínea em relação aos parâmetros basais (> $20 \mathrm{mmHg}$ ); Apreensão;

Hipervigilância das atividades; Olhos arregalados

Aumento moderado da pressão sanguínea em relação aos parâmetros basais ( $<20 \mathrm{mmHg}$ )

Sensação expressa de necessidade de oxigênio aumentada

Respiração ofegante

Uso total da musculatura acessória da respiração; Leve diaforese; Fadiga; Sensação de calor

9

8

7

6

5

4

2

Respiração abdominal paradoxal; Incapacidade de responder às solicitações verbais; Perguntas sobre possível funcionamento inadequado do aparelho

Cianose, Diaforese profusa, Nível de consciência diminuído, Respiração descoordenada com o ventilador, Diaforese, Incapacidade de cooperar, Mudanças na coloração e Palidez
Os resultados dispostos na Tabela 3 indicam o valor de acurácia das características definidoras para a predição do insucesso do desmame ventilatório e demonstraram que as mais sensíveis, isto é, aquelas com percentual maior de $70 \%$ foram: a frequência respiratória aumenta de forma significativa em relação aos parâmetros basais (grave) e aumento da frequência respiratória em relação aos parâmetros basais ( $<5$ respirações/min) (moderada).

Por seu turno, as características definidoras mais específicas verificadas na amostra foram: agitação (grave), deterioração nos gases sanguíneos arteriais em relação aos parâmetros basais (grave) e uso moderado da musculatura acessória da respiração (moderada).

Tanto os valores preditivos positivos quanto os negativos indicam a validade das mencionadas características definidoras. Contudo, indicam mais fortemente que a ausência da agitação, deterioração dos gases sanguíneos e o uso da musculatura acessória sejam um bom indicador evolutivo do sucesso da tentativa do desmame.

As razões de chances e de verossimiIhança positiva de valores mais elevados para a agitação, deterioração nos gases sanguíneos arteriais em relação aos parâmetros basais e uso moderado da musculatura acessória da respiração indicam um maior valor clínico de acurácia.

$\mathrm{Na}$ Tabela 3 estão indicadas apenas as características definidoras propostas pela NANDA - Internacional em que os valores mínimos calculados das razões de chance diagnósticas (RCD) no intervalo de confiança (IC) de 95\% superaram 1.0. As demais não alcançaram valores mínimos de RCD maiores do que 1.0, levando ao seu descarte como elementos de acurácia.

Como verificado apenas três das 15 características definidoras graves da NANDA-Internacional e duas moderadas em 14 possíveis alcançaram uma razão de chances no intervalo mínimo maior do que 1.0 e passaram no teste de significância. Nenhuma das oito características definidoras apontadas como leves pela NANDA-Internacional alcançou significância estatística.

As acurácias ou eficiências das características como indicadores de teste para o desfecho foram em torno de $70 \%$. 
Tabela 3 - Medidas de acurácia das características definidoras para indicar o sucesso/insucesso da tentativa de desmame, Rio de Janeiro-RJ, 2014

\begin{tabular}{|c|c|c|c|c|c|c|c|c|}
\hline $\begin{array}{l}\text { Características Definidoras } \\
\text { (tipo conforme a gravidade) }\end{array}$ & SE (\%) & ES (\%) & $\begin{array}{c}\mathrm{VP}+ \\
(\%)\end{array}$ & $\begin{array}{l}\text { VP- } \\
(\%)\end{array}$ & $\mathbf{R V}+$ & RV- & RCD* & $\begin{array}{l}\mathrm{EF} \\
(\%)\end{array}$ \\
\hline $\begin{array}{l}\text { 1. A frequência respiratória } \\
\text { aumenta de forma significativa } \\
\text { em relação aos parâmetros basais } \\
\text { (grave) }\end{array}$ & 83.3 & 55.0 & 62.5 & 78.6 & 1.8 & 0.3 & $6.11(1.33-27.96)$ & 68.4 \\
\hline $\begin{array}{l}\text { 2. Aumento da frequência } \\
\text { respiratória em relação } \\
\text { aos parâmetros basais }(<5 \\
\text { respirações/min) (moderada) }\end{array}$ & 72.2 & 65.0 & 65.0 & 72.2 & 2.1 & 0.4 & $4.83(1.21-19.21)$ & 68.4 \\
\hline 3. Agitação (grave) & 38.9 & 95.0 & 87.5 & 63.3 & 7.8 & 0.6 & $12.09(1.31-111.65)$ & 68.4 \\
\hline $\begin{array}{l}\text { 4. Deterioração nos gases } \\
\text { sanguíneos arteriais em relação } \\
\text { aos parâmetros basais (grave) }\end{array}$ & 38.9 & 100.0 & 100.0 & 64.1 & - & 0.6 & $26.73(1.40-512.10)$ & 71.0 \\
\hline $\begin{array}{l}\text { 5. Uso moderado da musculatura } \\
\text { acessória da respiração } \\
\text { (moderada) }\end{array}$ & 44.4 & 90.0 & 80.0 & 64.3 & 4.4 & 0.6 & $7.20(1.27-40.68)$ & 68.4 \\
\hline
\end{tabular}

* Razões de chances estatisticamente significativas, pois, o intervalo de confiança supera 1.0. Entre parênteses os intervalos mínimos e máximos para uma confiança de $95 \%$.

Notas: sensibilidade (SE), especificidade (ES), valores preditivos positivos (VP+) e negativos (VP-), razão de verossimilhança positiva (RV+) e negativa (RV-); razão de chances diagnóstica (RCD) e acurácia ou eficiência (EF).

\section{DISCUSSÃO}

Para um desmame ventilatório os pacientes são avaliados sobre suas capacidades para respirar espontaneamente. Em muitas unidades de terapia intensiva as avaliações são feitas por enfermeiros ou terapeutas respiratórios ${ }^{(2)}$ ou as informações da equipe de enfermagem são consideradas na decisão sobre o sucesso e insucesso das tentativas. Assim, é de alta relevância que enfermeiros possam julgar de forma precoce e acurada os desmames.

Os resultados do estudo indicam que em $47,37 \%$ das tentativas de desmame a interrupção se deu por julgamento clínico sobre uma ou duas evidências associadas de sinais ou sintomas anormais, especialmente ligados a respiração, pressão arterial, e inquietação. A base da decisão seria a orientação do protocolo institucional. Assim, a taxa de insucesso relativamente alta verificada no estudo pode estar relacionada a fatores como a experiência profissional; e a fatores dos pacientes como a comorbidade, a clínica e a idade maior ou igual a 65 anos $^{(2)}$.

Recomendações apontam que o sucesso da tentativa de desmame deve considerar a respiração espontânea ao menos por 30 minutos sem ou com pouco suporte ventilatório julgada nos seguintes indicadores de insucesso: frequência respiratória maior que 35 irpm por mais de 05 minutos; saturação inferior a $90 \%$; frequência cardíaca maior do que 140 batimentos/minuto; mudança maior do que $20 \%$ da linha de base; valores de pressão sistólica maiores do que $180 \mathrm{mmHg}$ ou menores do que $90 \mathrm{mmHg}$; aumento da ansiedade e diaforese ${ }^{(9-11)}$. Tais convergiram para alguns dos motivos de interrupção do desmame na presente pesquisa.
Quando se correlaciona o número de motivos alegados para interrupção com número de características definidoras observadas, há uma discrepância. Se, por um lado, as enfermeiras indicavam um ou dois motivos para interrupção do desmame, nesses mesmos eventos a observação contínua da pesquisa havia revelado uma mediana de 9,5 características definidoras da RDDV, sendo o limite mínimo de 06 ocorrências (Tabela 01). Interpretando tais achados é possível defender o argumento de que as características definidoras ampliam o rol disponível de evidências a se considerar em uma situação de insucesso do desmame.

Nos eventos de sucesso e insucesso as diferenças entre as medianas do número de características definidoras foram significativas para as graves e moderadas, indicando o valor do número mínimo de evidências para diagnosticar. Assim, seis características definidoras, especialmente entre graves e moderadas, indicariam o insucesso do desmame.

Os resultados referentes ao número de características definidoras ampliam os critérios para observação do desmame ventilatório, e quando associados às medidas de acurácia podem facilitar a identificação das situações de insucesso com maior precisão.

No que concerne ao indicador "a frequência respiratória aumenta de forma significativa em relação aos parâmetros basais", um estudo desenvolvido com 166 pacientes internados em terapia intensiva no Brasil verificou que o aumento da frequência respiratória para mais de 24 irpm é um excelente preditor do insucesso do desmame (Sensibilidade 100\%, Especificidade $85 \%$, e razão de verossimilhança positiva de 6,68$)^{(12)}$.

Para a característica "deterioração nos gases sanguíneos arteriais em relação aos parâmetros basais", que se mostrou 
altamente sensível, pode-se realizar ao menos dois procedimentos de avaliação, sendo os mais acessíveis e menos invasivos: (1) monitoração da saturação de oxigênio (oximetria de pulso) e (2) gás carbônico exalado (capnografia). Já a monitoração dos gases arteriais por gasometria arterial é o que requer mais invasão e tempo para realização, ainda que tenha maior valor de precisão. Estudo realizado com uma amostra de 73 pacientes de terapia intensiva na Escócia, verificou que apenas a medição do íon hidrogênio $\left(\mathrm{H}^{+}\right)$no sangue foi evidência diferente entre os grupos que atingiram sucesso no desmame e os que não atingiram ${ }^{(13)}$. Nesse estudo a indicação da deterioração dos gases (expresso na queda da oximetria) foi o motivo mais alegado para a interrupção do desmame.

A agitação não consta como critério institucional do protocolo de interrupção da tentativa de desmame ventilatório, porém, foi frequente nos eventos que culminaram com o insucesso, e raro nos bem-sucedidos. Considerando a alta especificidade $(95 \%)$ e razão de chances verificadas no estudo, a agitação deve ser monitorada em pacientes em desmame ventilatório, pois sua ocorrência nem sempre é frequente, porém, quando presente, relaciona-se fortemente ao fracasso do desmame.

Considerando as evidências dessa pesquisa e de outros estudos, defende-se que a precisão no diagnóstico pode ser alcançada mais facilmente pela reunião dos seguintes critérios no julgamento diagnóstico: (a) o número mínimo de características definidoras identificadas (Tabela 1) e (b) a medida de acurácia das categorias verificadas (Tabela 3). Quando aplicável, ainda considerar um terceiro critério: a temporalidade das características definidoras ${ }^{(5)}$.

Os achados indicam o valor de cinco indicadores clínicos (características definidoras), apontando a representatividade individual para o fenômeno diagnóstico que é julgado e colaborando com a validação do diagnóstico de enfermagem ${ }^{(14)}$.

No estudo realizado para investigação da temporalidade das características definidoras da RDDV verificou-se que por volta de 30 minutos (tempo da primeira avaliação da tentativa) apenas 33\% das características definidoras de uma RDDV haviam se manifestado(5).

Baseado nos achados do mencionado estudo(5), nas medidas de acurácia e de relação das medianas de ocorrência pode se argumentar ser menos comum a manifestação de características definidoras graves e moderadas antes de 30 minutos, exceto em situações em que o fracasso transcorrer rápida e insidiosamente. De fato, as características leves se concentram nesse intervalo e poderiam servir ao diagnosticador como um alerta para a evolução já que nenhuma delas alcançou valor de predição suficiente no presente estudo.

Por outro lado, para os eventos típicos, ou seja, os que evoluem mais lentamente, as características definidoras identificadas como as de maior acurácia se manifestam no tempo compreendido entre 40 a 99 minutos, considerando-se os valores de suas medianas ${ }^{(5)}$.

\section{CONCLUSÕES}

Conclusões podem ser apresentadas a partir dos achados do presente estudo e da interpretação da literatura. Houve validade das características definidoras da NANDA - Internacional, sendo que cinco delas podem ser bons indicadores de acurácia para avaliar as tentativas de desmame. Ainda, houve coerência na relação entre o número de características definidoras e os desfechos de sucesso/insucesso, isso para as graves e moderadas.

A presente pesquisa não investigou o julgamento diagnóstico de enfermeiras sobre a RDDV, entretanto, pode se supor que a precisão diagnóstica seja ampliada com a conjugação das variáveis: número de evidências (características definidoras), poder preditivo e distribuição temporal das características.

Ainda que contribua com originalidade e relevância ao conhecimento da acurácia do diagnóstico de enfermagem em sua aplicação como instrumento clínico da profissão, o estudo possui limitações: o reduzido número de pacientes e eventos, e o método de acompanhamento à beira do leito, que pode ser longo e exaustivo, possibilitando erros de omissão na coleta de dados. Além disso, não foram avaliadas variáveis de confusão ou de modificação de efeito.

Por fim, recomenda-se a replicação do estudo considerando uma população mais heterogênea nos critérios de idade e de situação clínica e cirúrgica.

\section{REFERÊNCIAS}

1. North American Nursing Diagnosis Association (NANDA international). Diagnósticos de Enfermagem: definições e classificações-2009-2011. Porto Alegre (RS): Artmed; 2010.

2. Damasceno MPCD, David CMN, Souza PCSP, Chiavone PA, Cardoso LTQ, Amaral JLG, et al. Ventilação mecânica no Brasil: aspectos epidemiológicos. Rev Bras Ter Intensiva [Internet]. 2006 [acesso em 16 de abril de 2014];18(3):219-28. Dísponível em: http://www.scielo.br/scielo.php?pid = S0 103-507X2006000300002\&script $=$ sci_arttext

3. McConville JF, Kress JP. Weaning patients from the ventilator. N Engl J Med [Internet]. 20126 [cited 2013 June
08];367:2233-9. Available from: http://www.nejm.org/ doi/full/10.1056/NEJMra1203367

4. Lunney M. Critical need to adress accuracy of nurse's diagnoses. Online J Issues Nurs [Internet]. 2008 [cited 2014 April 16];13(1):[about 15 p.]. Available from: http://nursingworld.org/MainMenuCategories/ANAMarketplace/ANAPeriodicals/OJIN/TableofContents/vol132008/No1Jan08/ ArticlePreviousTopic/AccuracyofNursesDiagnoses.html

5. Cerqueira FA, Brandão MAG, Mattos VZ, Castellões TMFW. Investigação da temporalidade das características definidoras do diagnóstico de resposta disfuncional ao desmame ventilatório. Esc Anna Nery Rev Enferm [Internet]. 2012 [acesso 
em 08 de junho de 2013];16(3):545-52. Disponível em: http://www.scielo.br/scielo.php?script=sci arttext\&pid $=$ S1414-81452012000300017\&lng $=$ en

6. Sousa VE, Lopes MVO, Araújo TL, Rolim IL, Nascimento RV, Oliveira TF. Clinical indicators of ineffective airway clearance for patients in the cardiac postoperative period. Eur J Cardiovasc Nurs [Internet]. 2013 [cited 2014 April 16];12(2):193-200. Available from: http://www.ncbi.nlm. nih.gov/pubmed/22531662

7. Lopes MVO, Silva VM, Araújo TL. Methods for establishing the accuracy of Clinical Indicators in Predicting Nursing Diagnoses. International J Nursing Knowledge [Internet]. 2012 [cited 2012 June 08];23(1):134-9. Available from: http://onlinelibrary.wiley.com/doi/10.1111/j.2047-30 95.2012.01213.x/abstract

8. Cavalcante TF, Araújo TL, Moreira RP, Guedes NG, Lopes MVO, Silva VM. Validação clínica do diagnóstico de enfermagem: risco de aspiração entre os pacientes que sofreram acidente vascular cerebral. Rev Latinoam Enferm [Internet]. 2013 [acesso em 08 de junho de 2013];21(no. esp.):250-8. Available from: http://www. scielo.br/scielo.php?script =sci_arttext\&pid = S010411692013000700031\&lng = en

9. III Consenso Brasileiro de Ventilação Mecânica: ventilação mecânica não invasiva com pressão positiva. J Bras Pneumol [Internet]. 2007 [acesso em 08 de junho de 2013];33(Suppl 2):S51-S70. Disponível em: http://www.jor-
naldepneumologia.com.br/PDF/Suple_151_47_3cap3.pdf

10. Esteban A, Alía I, Ibañez J, Benito S, Tobin MJ. Modes of mechanical ventilation and weaning: a national survey of spanish hospitals. The Spanish Lung Failure Collaborative Group. Chest [Internet] 1994 [cited 2012 June 08];106(4):1188-93. Available from: http://www.ncbi. nlm.nih.gov/pubmed/7924494

11. Esteban A, Ferguson ND, Meade MO, Frutos-Vivar F, Apezteguia $C$, Brochard $L$, et al. Evolution of mechanical ventilation in response to clinical research. Am J Respir Crit Care Med. 2008;177(2):170-7.

12. Lima EJS. Respiratory rate as a predictor of weaning failure from mechanical ventilation. Rev Bras Anestesiol [Internet]. 2013 [cited 2014 July 04];63(1):7-12. Available from: http://www.scielo.br/scielo.php?script $=$ sci arttext\&pid $=$ S0034-70942013000100001\&lng $=$ en

13. Monaco F, Drummond GB, Ramsay P, Servillo G, Walsh TS. Do simple ventilation and gas exchange measurements predict early successful weaning from respiratory support in unselected general intensive care patients? $\mathrm{Br}$ J Anaesth. 2010;105(3):326-33.

14. Lopes MVO, Silva VM, Araújo TL. Validação de diagnósticos de enfermagem: desafios e alternativas. Rev Bras Enferm [Internet]. 2013 [acesso em 17 de março de 2014];66(5):649-55. Disponível em: http://www. scielo.br/scielo.php? script = sci_arttext\&pid = S0034$-71672013000500002 \& \operatorname{lng}=$ en 